\title{
Empatía y arte en estudiantes de medicina
}

\author{
Teresa Guilera
}

Introducción. La empatía es la habilidad de identificar las emociones y pensamientos de otra persona y responder con una emoción adecuada. Las personas creativas son capaces de percibir las emociones e identificarlas con precisión. La empatía médica podría considerarse un arte que se puede aprender con las diversas manifestaciones artísticas.

Objetivo. Analizar la relación entre empatía y arte en estudiantes de medicina.

Sujetos y métodos. 110 voluntarios cumplimentaron un cuestionario de preguntas relacionadas con la expresión artística y el hábito de lectura no médica, y de tres escalas de empatía: Jefferson Scale of Physician Empathy, Empathy Quotient e Interpersonal Reactivity Index, que consta de cuatro subescalas: perspective taking, fantasy scale, empathetic concern y personal distress.

Resultados. Un 77,4\% eran mujeres, un 69,1\% habían realizado algún tipo de expresión artística y un 45,5\% leían de forma habitual literatura no médica. La percepción empática $(p=0,026)$ y la expresión artística $(p=0,007)$ fueron mayores en mujeres. Solamente los estudiantes que habían realizado algún tipo de expresión artística mostraron una mayor puntuación en la subescala de fantasía $(p=0,015)$.

Conclusiones. La habilidad para ponerse en situaciones ficticias está más desarrollada en personas con tendencias artísticas. Sería interesante fomentar el hábito de lectura en estudiantes de medicina y que pudieran mantener alguna disciplina artística. De esta manera, se estimularía la creatividad, la apreciación artística y las habilidades empáticas, tan necesarias en el futuro profesional de los estudiantes de medicina.

Palabras clave. Arte. Creatividad. Empatía. Estudiantes de medicina. Literatura.

\section{Empathy and arts in medical students}

Introduction. Empathy is the ability to identify and understand the thoughts and feelings of others and to respond with appropriate emotions. Creative people are able to perceive emotions and identify them accurately. Medical empathy could be considered an art that can be learned with different artistic expressions.

Aim. To analyze the relationship between empathy and art in medical students.

Subjects and methods. 110 volunteers completed a questionnaire with questions related to artistic expression and the habit of non-medical reading. It was used three empathy scales: Jefferson Scale of Physician Empathy, Empathy Quotient and Interpersonal Reactivity Index with four subscales: perspective taking, fantasy, empathetic concern and personal distress.

Results. $77.4 \%$ were women, $69.1 \%$ had done some artistic expression and $45.5 \%$ regularly read non-medical literature. Empathic concern $(p=0.026)$ and artistic expression $(p=0.007)$ were higher in women. Only the students who had done some artistic expression showed a higher score on fantasy subscale $(p=0.015)$.

Conclusions. The ability to put yourself in fictitious situations is more developed in people with artistic preferences. It would be interesting to encourage the habit of reading in medical students, and that they could maintain some artistic discipline. In this way, creativity, artistic appreciation and empathic skills, so necessary in the professional future of medical students, would be stimulated.

Key words. Arts. Creativity. Empathy. Literature. Medical students.

\section{Introducción}

La empatía médica es una habilidad eminentemente cognitiva que implica la comprensión de las experiencias, inquietudes y perspectivas del paciente, combinadas con la capacidad de comunicar esta comprensión y la intención de ayudar [1]. Se define empatía cognitiva como la capacidad de reconocer lo que el otro piensa o siente [2], y la empatía afectiva, como la capacidad de sentir una emoción apropiada en respuesta a los pensamientos o sentimientos del otro $[3,4]$.
Institut de Recerca Biomèdica de Lleida (IRBLleida). Servicio de Psiquiatría. Hospital Universitari de Santa Maria. Gestió de Serveis Sanitaris. Lleida, España.

Correspondencia:

Dra. Teresa Guilera Lladós. Institut de Recerca Biomèdica de Lleida (IRBLleida). Servicio de Psiquiatría. Hospital Universitari de Santa Maria. Gestió de Serveis Sanitaris. Avda. Rovira Roure, 44. E-25198 Lleida.

E-mail:

tguilera37@gmail.com

Agradecimientos: Dra. Iolanda Batalla y Dr. Jorge Soler-González.

Recibido: 14.03.18.

Aceptado: 20.03.18.

Conflicto de intereses: No declarado.

Competing interests: None declared.

(c) 2018 FEM 
Tabla I. Análisis descriptivo de la muestra $(n=110)$.

\begin{tabular}{|c|c|c|c|}
\hline \multirow{9}{*}{$\begin{array}{l}\text { Variables } \\
\text { sociobiográficas }\end{array}$} & \multicolumn{2}{|l|}{ Edad (años) } & $22(21-23)$ \\
\hline & \multirow{2}{*}{ Sexo } & Hombre & $26(23,6 \%)$ \\
\hline & & Mujer & $84(76,4 \%)$ \\
\hline & \multirow{2}{*}{ Curso } & Primero a tercero & $41(37,3 \%)$ \\
\hline & & Cuarto a sexto & $69(62,7 \%)$ \\
\hline & \multirow{2}{*}{$\begin{array}{l}\text { Preferencia } \\
\text { de especialidad }\end{array}$} & Orientada a las personas & $80(72,7 \%)$ \\
\hline & & Orientada a la tecnología & $30(27,3 \%)$ \\
\hline & \multicolumn{2}{|l|}{ Expresión artística } & $76(69,1 \%)$ \\
\hline & \multicolumn{2}{|c|}{ Hábito de lectura de ficción } & $50(45,5 \%)$ \\
\hline \multirow{10}{*}{$\begin{array}{l}\text { Variables psicométricas } \\
\text { (escalas de empatía) }\end{array}$} & \multicolumn{2}{|c|}{ Jefferson Scale of Physician Empathy } & $120,5(110,7-130)$ \\
\hline & \multirow{4}{*}{$\begin{array}{l}\text { Interpersonal } \\
\text { Reactivity } \\
\text { Index (IRI) }\end{array}$} & IRI-Perspective taking & $18,5(16-21)$ \\
\hline & & IRI-Fantasy scale & $18(14-24)$ \\
\hline & & IRI-Empathetic concern & $23(20-24)$ \\
\hline & & IRI-Personal distress & $8(5-11,2)$ \\
\hline & \multicolumn{2}{|l|}{ Empathy Quotient } & $49(41-57)$ \\
\hline & \multicolumn{2}{|l|}{ Empatía baja } & $5(4,5 \%)$ \\
\hline & \multicolumn{2}{|l|}{ Empatía media } & $61(55,5 \%)$ \\
\hline & \multicolumn{2}{|c|}{ Empatía por encima de la media } & $36(32,7 \%)$ \\
\hline & \multicolumn{2}{|c|}{ Empatía muy alta } & $8(7,3 \%)$ \\
\hline
\end{tabular}

Las variables se describen con la mediana (rango intercuartílico), y las variables cualitativas, con el número y porcentaje. ternura ante el malestar del otro, y personal distress (IRI-PD), que mide los sentimientos de ansiedad y malestar que el sujeto manifiesta al observar las experiencias negativas de los demás. Empathy Quotient (EQ) es una escala que se basa en el modelo psicológico propuesto por Baron-Cohen, y que permite la clasificación de la empatía en cuatro categorías (baja, media, por encima de la media y muy alta), facilitando la comparación entre grupos.

Algunos estudios muestran diferencias de sexo en estudiantes de medicina tanto en la JSPE [5,10-21] y algunas subescalas del IRI [9,22], como en el EQ [2].

La empatía desempeña un papel muy importante en el acto creativo, el cual necesita de la imaginación y la fantasía. A partir de los conocimientos adquiridos y de las experiencias vividas en el pasado, la imaginación permite anticipar el futuro y adoptar el punto de vista de otra persona [23]. Esta habilidad puede ser muy útil en la práctica médica en la que, a partir de los datos tanto objetivos como subjetivos, se debe establecer un plan terapéutico con la finalidad de ayudar al paciente.

Las obras de arte transmiten emociones y forman parte del proceso creativo. Las personas creativas son capaces de percibir las emociones e identificarlas con precisión. Según algunos autores, las personas creativas se distinguen de las demás por estar abiertas a vivir nuevas experiencias, ser curiosas y poco convencionales [24-28] y, normalmente, desde su infancia tienen intereses artísticos e intelectuales [29].

Estudios publicados reflexionan si la medicina debe considerarse una ciencia o un arte [30] y la implicación del arte en la formación médica [31,32]. La empatía médica podría considerarse un arte que necesita ser aprendido y no se consigue solamente con clases teóricas o guías prácticas. Según algunos autores, la empatía también se puede aprender con las diversas manifestaciones del arte, entre ellas, la literatura y el cine [33].

El objetivo de este trabajo es analizar la relación entre la empatía y el arte (expresión artística y hábito de lectura de literatura no médica) en estudiantes de medicina.

\section{Sujetos y métodos}

La población a estudio la formaron estudiantes de medicina: 110 voluntarios sanos aceptaron, mediante consentimiento informado, participar en este estudio observacional transversal, realizado durante el curso académico 2015-2016. Los alumnos cumplimentaron el cuestionario en el que se recogieron 
variables sociobiográficas y psicométricas. Se utilizaron tres instrumentos de medida de la empatía:

- JSPE. Consta de 20 ítems, con un rango de puntuaciones de 20-140. Mide la empatía específicamente en estudiantes de medicina y médicos en el contexto del cuidado del paciente [5]. Se ha traducido del inglés y se ha validado en castellano [34].

- IRI. Consta de cuatro subescalas de siete ítems cada una, con un rango de puntuaciones de 0-28. Dos subescalas miden la empatía cognitiva (IRIPT e IRI-FS), y dos, la empatía afectiva (IRI-EC e IRI-PD) $[6,35]$.

- EQ. Consta de 60 ítems, 40 de empatía y 20 de control. Rango de puntuaciones de 0-80. Mide la empatía cognitiva y afectiva en adultos. Permite clasificar la empatía en baja (0-32), media (33-52), por encima de la media (53-63) y muy alta (64-80). La versión no validada en castellano se obtiene a través de la web http://espectroautista.info/tests/ espectro-autista/adultos/EQ. Se dispone del consentimiento del autor del EQ [2] para su uso.

La implicación con las artes en estudiantes de medicina se valoró mediante dos preguntas cerradas con respuestas sí/no: ‘ ¿realiza o ha realizado algún tipo de expresión artística (música, danza, pintura, escritura...)?, ‘'lee de forma habitual literatura no médica?'.

En el análisis descriptivo de los datos se obtuvieron porcentajes para las variables cualitativas. Las escalas de empatía mostraron una distribución no normal, evaluada mediante la prueba de ShapiroWilks, para las cuales se obtuvo la mediana y el rango intercuartílico. Se analizaron las diferencias en las escalas de empatía en toda la población de estudio, estratificadas por sexo, expresión artística y hábito de lectura de literatura de ficción, mediante la prueba $U$ de Mann-Whitney. Se utilizó la prueba exacta de Fisher para las variables cualitativas. Todas las pruebas fueron bilaterales, estableciendo el nivel de significación en 0,05. Todos los análisis se realizaron con el programa estadístico SPSS.

Este proyecto fue aprobado por el Comité de Ética de Investigación Clínica CEIC-1515.

\section{Resultados}

La tabla I refleja el análisis descriptivo de la muestra, y la tabla II, las diferencias en empatía y arte en función del sexo. Los resultados mostraron diferencias significativas entre sexos en la subescala IRI-EC $(p=0,026)$, siendo superior en mujeres. El porcentaje de mujeres que habían realizado algún tipo de
Tabla II. Empatía y arte en función del sexo.

\begin{tabular}{|c|c|c|c|c|c|}
\hline & & & Hombres $(n=26)$ & Mujeres $(n=84)$ & $p$ \\
\hline \multirow{6}{*}{ Empatía } & \multicolumn{2}{|c|}{ Jefferson Scale of Physician Empathy } & $120,5(111-128)$ & $120,5(110,2-130)$ & $0,657^{a}$ \\
\hline & \multirow{4}{*}{$\begin{array}{l}\text { Interpersonal } \\
\text { Reactivity } \\
\text { Index (IRI) }\end{array}$} & IRI-Perspective taking & $18(16-21,25)$ & $19(16-21)$ & $0,748^{a}$ \\
\hline & & IRI-Fantasy scale & $17(11-22,75)$ & $18(15-24)$ & $0,203^{a}$ \\
\hline & & IRI-Empathetic concern & $20(18-24)$ & $23(21-24,7)$ & $0,026^{a}$ \\
\hline & & IRI-Personal distress & $6,5(4-10,5)$ & $8(5-11,7)$ & $0,387^{a}$ \\
\hline & \multicolumn{2}{|c|}{ Empathy Quotient } & $46,5(40,7-56)$ & $50(43-57,7)$ & $0,402^{a}$ \\
\hline \multirow{2}{*}{ Arte } & \multicolumn{2}{|c|}{ Expresión artística } & $12(46,2 \%)$ & $64(76,2 \%)$ & $0,007^{b}$ \\
\hline & \multicolumn{2}{|c|}{ Literatura no médica } & $12(46,2 \%)$ & $38(45,2 \%)$ & $1^{\mathrm{b}}$ \\
\hline
\end{tabular}

Las variables se describen con la mediana (rango intercuartílico), y las variables cualitativas, con el número y porcentaje. a Prueba $U$ de Mann-Whitney para las variables cuantitativas; ${ }^{b}$ Prueba exacta de Fisher para las variables cualitativas.

expresión artística fue mayor, con una diferencia estadísticamente significativa $(p=0,007)$, mientras que el hábito de lectura de ficción no mostró diferencias entre sexos.

La tabla III muestra la relación entre las diferentes escalas de empatía y el arte. Solamente los estudiantes que habían realizado algún tipo de expresión artística mostraron una mayor puntuación en la subescala IRI-FS, siendo esta diferencia estadísticamente significativa $(p=0,015)$.

\section{Discusión}

Este estudio analiza la relación entre distintas escalas de empatía y arte en estudiantes de medicina. Respecto a estudios previos, en nuestra muestra, tenían puntuaciones más altas de empatía en las escalas JSPE $[1,12,13,16,36-40]$ y EQ [41] y en las subescalas IRI-FS $[6,22]$ e IRI-EC [17,22,41]. Estas diferencias podrían relacionarse, según han explicado otros autores, con la variabilidad intercultural entre diferentes países [42].

La utilización en nuestro estudio de tres escalas de empatía que complementan diferentes aspectos de la empatía cognitiva y afectiva ha permitido observar diferencias de sexo en la subescala IRI-EC. Así mismo, se observa que son más las mujeres que han realizado algún tipo de expresión artística, mientras que no se aprecian diferencias de sexo en el hábito de la lectura. Es decir, en mujeres se observa una 
Tabla III. Empatía en función de la expresión artística y el hábito de literatura no médica.

\begin{tabular}{|c|c|c|c|c|c|c|c|}
\hline & & \multicolumn{3}{|c|}{ Expresión artística } & \multicolumn{3}{|c|}{ Literatura no médica } \\
\hline & & Sí $(n=76)$ & No $(n=34)$ & $p^{\text {a }}$ & Sí $(n=50)$ & No $(n=60)$ & $p^{\text {a }}$ \\
\hline \multicolumn{2}{|c|}{ Jefferson Scale of Physician Empathy } & $121(111,2-130)$ & $120(108,7-128)$ & 0,584 & $120(108-126)$ & $124,5(113-131)$ & 0.058 \\
\hline \multirow{4}{*}{$\begin{array}{l}\text { Interpersonal } \\
\text { Reactivity } \\
\text { Index (IRI) }\end{array}$} & IRI-Perspective taking & $19(16,2-21,7)$ & $17(15,7-20)$ & 0,067 & $18(15,7-22)$ & $19(17-21)$ & 0,634 \\
\hline & IRI-Fantasy scale & $18,5(16-24)$ & $15,5(12-22)$ & 0,015 & $18(15-25)$ & $18(14-22)$ & 0,227 \\
\hline & IRI-Empathetic concern & $23(20-24,7)$ & $22,5(19-24)$ & 0,563 & $22,5(20-24,2)$ & $23(20-24)$ & 0,856 \\
\hline & IRI-Personal distress & $7,5(5-12)$ & $8(5-10)$ & 0,907 & $7(4,7-12)$ & $8(5-11)$ & 0,736 \\
\hline \multicolumn{2}{|c|}{ Empathy Quotient } & $51(42,2-58,7)$ & $45(41-51,5)$ & 0,089 & $48,5(40-58,2)$ & $49(43,2-56,7)$ & 0,701 \\
\hline
\end{tabular}

Las variables se describen con la mediana (rango intercuartílico). a Prueba $U$ de Mann-Whitney.

mayor empatía afectiva, tienen mayor capacidad para sentir lo que el otro siente y posiblemente mayor sensibilidad artística. La muestra del estudio está formada mayoritariamente por mujeres, aunque ello es el reflejo del alto porcentaje de mujeres presentes en los estudios de medicina. Los resultados también muestran que la empatía cognitiva y la habilidad para ponerse en situaciones ficticias están más desarrolladas en los estudiantes con tendencias artísticas. Por lo tanto, tienen facilidad para ponerse en la piel del personaje del cine o de la literatura e identificarse con él. Aunque la mayoría de los estudiantes mostraban un buen nivel de empatía, es importante tener en cuenta aquellos con valores extremos. A los estudiantes con bajos niveles de empatía les cuesta identificar las emociones de los demás porque no las entienden, mientras que en los alumnos con un nivel de empatía muy alto, su extrema sensibilidad les genera ansiedad y conductas de evitación social. Por tanto, en los valores extremos de empatía, la literatura de ficción permitiría ponerse en el lugar del otro y experimentar lo que los personajes de ficción sienten, pero en un contexto emocionalmente seguro [43]. Sentir miedo y ansiedad no es compatible con la empatía ni con el acto creativo, ya que crear implica mirar hacia fuera, no hacia uno mismo. Por este motivo, sería interesante enseñar, desde las universidades, a no estar tan centrados en uno mismo, aprender a mirar hacia fuera y a disfrutar del arte. De esta manera podrán aprender de las experiencias propias y a gestionarlas sin que las emociones generen malestar.

Las intervenciones psicoeducativas planteadas en estudios previos para mejorar la empatía se basan fundamentalmente en fomentar la toma de perspectiva y disminuir el malestar que generan las relaciones sociales [44]. El contacto con el arte, tanto desde el punto de vista de su apreciación como de su creación, puede ser una poderosa herramienta de enseñanza para mejorar las habilidades empáticas y la creatividad. Sin embargo, la creatividad precisa una actitud abierta, receptiva y no tener miedo a sentir. Aunque los estudiantes de medicina no tengan la experiencia ni la sabiduría que conlleva el paso de los años, poseen una imaginación sin límites que debería preservarse. Durante los estudios universitarios no disponen de tiempo para leer ni para las aficiones artísticas que iniciaron durante la infancia y la adolescencia, y muchos de ellos las abandonan. Teniendo en cuenta los resultados de este estudio, sería interesante que los estudiantes de medicina pudieran mantener alguna de las disciplinas artísticas que iniciaron en etapas anteriores, así como fomentar el hábito de lectura no médica. Aunque pueda ser una utopía pensar que todos los alumnos lleguen a desarrollar habilidades artísticas, sí es posible que aprendan a apreciar y a disfrutar del arte. De esta manera, se estimularía la creatividad y las habilidades empáticas, tan necesarias en el futuro profesional de los estudiantes de medicina.

\section{Bibliografía}

1. Hojat M. Ten approaches for enhancing empathy in health and human services cultures. J Health Hum Serv Adm 2009; 31: 412-50.

2. Baron-Cohen S, Wheelwright S. The Empathy Quotient: an investigation of adults with Asperger syndrome or high functioning autism, and normal sex differences. J Autism Dev Disord 2004; 34: 163-75. 
3. Mehrabian A, Eipstein N. A measure of emotional empathy J Pers 1972; 40: 525-43.

4. Cox CL, Uddin LQ, Di Martino A, Castellanos FX, Milham MP, Kelly $\mathrm{C}$. The balance between feeling and knowing: affective and cognitive empathy are reflected in the brain's intrinsic functional dynamics. Soc Cogn Affect Neurosci 2012; 7: 727-37.

5. Hojat M, Gonnella JS, Nasca TJ, Mangione S, Vergare M, Magee M. Physician empathy: definition, components, measurement, and relationship to gender and specialty. Am J Psychiatry 2002; 159: 1563-9.

6. Davis MH. Measuring individual differences in empathy: evidence for a multidimensional approach. J Pers Soc Psychol 1983; 44: 113-26.

7. Pérez-Albéniz A. Adaptación de Interpersonal Reactivity Index (IRI) al español. Psicothema 2003; 15: 267-72.

8. Mestre-Escrivá V, Frías-Navarro MD, Samper-García P. La medida de la empatía: análisis del Interpersonal Reactivity Index. Psicothema 2004; 16: 255-60.

9. Neumann M, Scheffer C, Tauschel D, Lutz G, Wirtz M, Edelhäuser F. Physician empathy: definition, outcome-relevance and its measurement in patient care and medical education. GMS Z Med Ausbild 2012; 29: Doc11.

10. Chen DC, Pahilan ME, Orlander JD. Comparing a selfadministered measure of empathy with observed behavior among medical students. J Gen Intern Med 2010; 25: 200-2.

11. Chen D, Lew R, Hershman W, Orlander J. A cross-sectional measurement of medical student empathy. J Gen Intern Med 2007; 22: 1434-8.

12. Chen DC, Kirshenbaum DS, Yan J, Kirshenbaum E, Aseltine $\mathrm{RH}$. Characterizing changes in student empathy throughout medical school. Med Teach 2012; 34: 305-11.

13. Kataoka HU, Koide N, Ochi K, Hojat M, Gonnella JS. Measurement of empathy among Japanese medical students: psychometrics and score differences by gender and level of medical education. Acad Med 2009; 84: 1192-7.

14. Kataoka HU, Koide N, Hojat M, Gonnella JS. Measurement and correlates of empathy among female Japanese physicians. BMC Med Educ 2012; 12: 48.

15. Tavakol S, Dennick R, Tavakol M. Psychometric properties and confirmatory factor analysis of the Jefferson Scale of Physician Empathy. BMC Med Educ 2011; 11: 54.

16. Magalhães E, Salgueira AP, Costa P, Costa MJ. Empathy in senior year and first year medical students: a cross-sectional study. BMC Med Educ 2011; 11: 52.

17. Quince TA, Parker RA, Wood DF, Benson JA. Stability of empathy among undergraduate medical students: a longitudinal study at one UK medical school. BMC Med Educ 2011; 11: 90.

18. Suh DH, Hong JS, Lee DH, Gonnella JS, Hojat M. The Jefferson Scale of Physician Empathy: a preliminary psychometric study and group comparisons in Korean physicians. Med Teach 2012; 34: e464-8.

19. Hasan S, Al-Sharqawi N, Dashti F, AbdulAziz M, Abdullah A Shukkur M, et al. Level of empathy among medical students in Kuwait University, Kuwait. Med Princ Pract 2013; 22: 385-9.

20. Hegazi I, Wilson I. Maintaining empathy in medical school: it is possible. Med Teach 2013; 35: 1002-8.

21. Santos MA, Grosseman S, Morelli TC, Giuliano ICB, Erdmann TR. Empathy differences by gender and specialty preference in medical students: a study in Brazil. Int J Med Educ 2016; 21: 153-7.

22. Grau A, Torán P, Zamora A, Quesada M, Carrión C, Vilert E, et al. Evaluación de la empatía en estudiantes de medicina. Educación Médica 2017; 18: 114-20.

23. Robinson K. Busca tu elemento. Aprende a ser creativo individual y colectivamente. Barcelona: Empresa Activa; 2012.

24. Feist GJ. The influence of personality on artistic and scientific creativity. New York: Cambridge University Press; 1999.
25. Ivcevic Z. Artistic and everyday creativity: an act-frequency approach. J Creat Behav 2007; 41: 271-90.

26. Ivcevic Z. Creativity map: toward the next generation of theories of creativity. Psychology of Aesthetics, Creativity, and the Arts 2009; 3: 17-21.

27. King LA, Walker LM, Broyles SJ. Creativity and the five-factor model. J Res Pers 1996; 30: 189-203.

28. McCrae RR. Creativity, divergent thinking, and openness to experience. J Pers Soc Psychol 1987; 52: 1258-65.

29. Helson R, Roberts B, Agronick G. Enduringness and change in creative personality and the prediction of occupational creativity. J Pers Soc Psychol 1995; 69: 1173-83.

30. Romero-Leguizamón CR. ¿Medicina: arte o ciencia? Una reflexión sobre las artes en la educación médica. Educación Médica 2017. doi: 10.1016/j.edumed.2017.04.005

31. Weisz GM, Albury WR. The medico-artistic phenomenon and its implications for medical education. Med Hypotheses 2010; 74: 169-73.

32. Saura-Carretero Z, López-Aragó M, López-Castellano A, Rodilla V. El arte como herramienta docente en medicina. Educación Médica 2017; doi: 10.1016/j.edumed.2017.10.004

33. Moreto GM, González-Blasco P, Janaudis MA, De Benedetto MAC. Modificación del nivel de empatía de estudiantes de medicina durante la graduación médica. SOBRAMFA Educación Médica \& Humanismo 2009; 619-25.

34. Alcorta A, Juan G, Guerrero FG, Tavitas SE, Rodríguez FJ, Mohammadreza L, et al. Validación de la escala de empatía médica de Jefferson en estudiantes de medicina mexicanos. Educ Med 2005; 28: 57-63.

35. Carrasco-Ortiz MA, Delgado-Egido B, Barbero-García MI, Holgado-Tello FP, Del Barrio-Gándara MV. Propiedades psicométricas del Interpersonal Reactivity Index (IRI) en población infantil y adolescente española. Psicothema 2011; 23: 824-31.

36. Loureiro J, Gonçalves-Pereira M, Trancas B, Caldas-deAlmeida JM, Castro-Caldas A. Empathy in the doctor-patient relationship as viewed by first-year medical students: data on validity and sensibility to change of the Jefferson measure in Portugal. Acta Med Port 2011; 24: 431-42.

37. Nunes P, Williams S, Sa B, Stevenson K. A study of empathy decline in students from five health disciplines during their first year of training. Int J Med Educ 2011; 2: 12-7.

38. Lim BT, Moriarty H, Huthwaite M, Gray L, Pullon S, Gallagher P. How well do medical students rate and communicate clinical empathy? Med Teach 2013; 35: e946-51.

39. Costa P, Alves R, Neto I, Marvão P, Portela M, Costa MJ. Associations between medical student empathy and personality: a multi-institutional study. PLoS One 2014; 9: e89254.

40. Youssef FF, Nunes P, Sa B, Williams S. An exploration of changes in cognitive and emotional empathy among medical students in the Caribbean. Int J Med Educ 2014; 5: 185-92.

41. Handford C, Lemon J, Grimm MC, Vollmer-Conna U. Empathy as a function of clinical exposure-reading emotion in the eyes. PLoS One 2013; 8: e65159.

42. Melchers MC, Li M, Haas BW, Reuter M, Bischoff L, Montag C. Similar personality patterns are associated with empathy in four different countries. Front Psychol 2016; 7: 290.

43. Guilera T, Batalla I, Soler-González J. Empatía y preferencia de especialidad en estudiantes de medicina. Estudio de seguimiento y feedback. Educación Médica 2017. doi: 10.1016/ j.edumed.2017.07.017.

44. Guilera T. Empatía en estudiantes de medicina: estudio psicométrico, biométrico, de evolución y propuestas de intervención psicoeducativa [tesis doctoral]. Lleida: Universitat de Lleida; 2017. 